\title{
Traduire
}

Revue française de la traduction

$223 \mid 2010$

Tribunal et théâtre, faites entrer le traducteur

\section{Scènes judiciaires et théâtrales ou Le traducteur serviteur de deux maitres}

\section{Geneviève Bégou}

\section{(2) OpenEdition}

\section{Journals}

Édition électronique

URL : http://journals.openedition.org/traduire/257

DOI : $10.4000 /$ traduire. 257

ISSN : 2272-9992

Éditeur

Société française des traducteurs

\section{Édition imprimée}

Date de publication : 15 décembre 2010

Pagination : 3-7

ISSN : 0395-773X

\section{Référence électronique}

Geneviève Bégou, « Scènes judiciaires et théâtrales ou Le traducteur serviteur de deux maîtres ", Traduire [En ligne], 223 | 2010, mis en ligne le 10 février 2014, consulté le 22 septembre 2020. URL : http:// journals.openedition.org/traduire/257 ; DOI : https://doi.org/10.4000/traduire.257 


\section{é d i to}

Geneviève Bégou

\section{Scènes judiciaires et théâtrales ou Le traducteur serviteur de deux maîtres}

\section{Acte I}

Où l'on voit, côté cour, tout le sérieux de la profession

En lever de rideau, une excellente présentation de la fonction d'expert traducteur et interprète (ETI) en France et une interrogation sur la spécificité de cette " exception française ". Parlant d'expérience et maniant subtilement l'humour et l'ironie, Évelyne Fusilier relève les particularités, paradoxes et lacunes du système. En effet, auxiliaire de justice et du service public (exerçant souvent dans ce dernier cas sous l'appellation simplifiée de " traducteur/interprète assermenté " ou " juré "), l'ETI est intégré au corps des " experts " de justice - une situation qui semble unique au monde. L'abondance de textes législatifs ou réglementaires définissant et précisant la qualité d'expert judiciaire, voire jalonnant tous les aspects de sa vie professionnelle, de la candidature à la rémunération, fait surtout apparaître toute l'extrême complexité de la profession et du contexte de son exercice. Paradoxes et lacunes peuvent avoir des conséquences juridiques graves. Un exemple parmi d'autres : la dénomination des personnes inscrites sur les listes d'experts est fixée de manière stricte (et l'usurpation de titre sévèrement punie) mais les critères d'inscription retenus par la cour ne sont pas clairement indiqués. Lors de son inscription, l'ETI prête serment. Mais la justice peut confier une " mission de constatation, de consultation et d'expertise " à un " expert ne figurant sur aucune liste " et un juge peut désigner " toute autre personne de [son] choix " (le cas échéant, notamment au pénal, dans l'urgence et la catégorie "interprétariat ", le premier locuteur disponible de la langue recherchée). Dans un cas comme dans l'autre, si l'on omet de faire prêter serment, la procédure peut être entièrement annulée, même des années plus tard. Preuve, peut-être, qu'en dépit du cadre strict établi par le législateur, "la Justice ne perçoit pas la fonction d'expert comme une profession "? 
Venant démentir la remarque un peu désabusée d'É. Fusilier, la contribution de Bernadette Anton Bensoussan est d'autant plus intéressante que l'auteur n'est ni traductrice, ni interprète mais magistrate. Amenée à s'intéresser aux missions des interprètes, dans le cadre de ses activités, elle a pu constater les problématiques liées à l'obligation ou la nécessité du recours à l'interprète ou au traducteur pour être en conformité avec les textes en vigueur. Se contenter des services d'un locuteur natif en lieu et place d'un ETI, quelles que soient les raisons (urgence, rareté d'un idiome) ? Mais peut-il y avoir un service minimum de traduction en justice? Le risque d'erreur judiciaire ne devrait-il pas obliger toutes les parties à rechercher la perfection de la traduction?

Devant la nécessité grandissante de faire appel aux " hommes (et femmes) de l'art ", l'auteur pousse les ETI à " reconquérir " leur place au sein de la procédure et tout d'abord, à se faire connaître ! Parmi les quelques pistes évoquées : la diffusion officielle des listes des ETI aux institutions qui requièrent leurs services (commissariats, cabinets d'instruction, tribunaux pour enfants - la liste est loin d'être exhaustive) et un contact direct avec ces mêmes institutions pour expliquer la mission des ETI, car on constate une grande ignorance du monde judiciaire sur ses indispensables " auxiliaires de justice ". Se faire connaître, donc, mais aussi, mais surtout reconnaître. L'ETI est un professionnel et doit faire valoir sa formation, sa spécificité et sa rigueur. Quel meilleur moyen pour faire barrage à l'incompétence, à l'usurpation de titre et (un comble !) au travail au noir ? Un changement de mentalité doit s'opérer de part et d'autre. À l'ETI de ne plus rester en retrait, de se faire respecter, et de s'imposer. À l'institution judiciaire de repenser sa collaboration avec l'interlocuteur qui l'accompagnera parfois tout au long d'une audience.

Complexité en-deçà du Rhin, limpidité au-delà ? Voire ! II ressort du tableau que brosse Natacha Dalügge-Momme des réglementations concernant l'assermentation en Allemagne que non seulement les termes " interprète " et " traducteur " ne sont pas protégés, permettant à " toute personne se croyant apte à assumer la fonction " de pouvoir "le faire sans entrave " (qu'en termes élégants ces choses-là sont dites !) mais en outre, aucune loi fédérale ne définit la profession du traducteur assermenté et les juges indépendants ont le droit d'assermenter une personne ad hoc avant une procédure. Le système fédéral allemand ajoute bien évidemment sa petite touche de complexité au tableau. Pionnier en matière de législation, de code de déontologie et d'examen d'aptitude des candidats, le Land de Hambourg fait école dans plusieurs nouveaux Länder, sans toutefois parvenir à étendre son système à tout le territoire national. Les appellations " traducteur assermenté " ou " interprète assermenté " diffèrent selon le statut et selon les Länder. L'absence de normalisation des tampons entraîne souvent des problèmes de transfert de documents d'un Land à l'autre. Décernée "à vie " (sauf dans deux Länder), l'assermentation relève de la compétence du Land, qui peut la retirer pour faute grave ou incapacité, mais la responsabilité des assermentés relève de la compétence de différents ministères, cours ou tribunaux. Aujourd'hui, les directives et la jurisprudence européennes changent la donne mais les lois sont encore loin d'être homogènes. Cependant, le fort inves- 
tissement des associations professionnelles et l'établissement d'un registre national des traducteurs et interprètes assermentés représentent une avancée importante.

Fin de ce premier acte " judiciaire " : Julia Lobato Patricio s'intéresse à la réalité professionnelle de la traduction assermentée en Espagne. Agrémenté d'exemples concrets, son panorama actuel du métier détaille les concepts de traduction juridique, judiciaire et assermentée, puis les compétences nécessaires et spécifiques : linguistiques, textuelles, extralinguistiques, psychophysiologiques, relationnelles et stratégiques - sans oublier la souplesse d'adaptation et l'esprit d'autocritique. Nonobstant la prédisposition de certains pour l'exercice, l'auteur souligne la nécessité d'une formation spécialisée. De récents décrets royaux ont modifié, d'une part la voie d'accès traditionnelle à la profession, aujourd'hui limitée au passage de l'examen annuel du ministère des Affaires étrangères et de la coopération, d'autre part la dénomination officielle : "l'interprète assermenté " est désormais " traducteur-interprète assermenté " puisque son travail concerne l'oral et l'écrit. Si l'Association professionnelle espagnole des traducteurs et interprètes a jeté les bases d'une normalisation des traductions assermentées, il reste encore beaucoup à faire dans ce domaine. Un vaste champ de recherches en méthodologie s'ouvre donc aux professionnels et étudiants du secteur.

\section{Acte II}

\section{Où l'on penserait (à tort) que la vie est plus simple côté jardin}

Changement de décor. Le second volet de notre cahier consacré au théâtre s'intéresse d'abord au surtitrage théâtral. Enseignant, chercheur et praticien, membre de la compagnie de théâtre universitaire toulousaine "Les Anachroniques ", Bernard Péran s'attache à cerner les spécificités d'une pratique assez récente qui, malgré son rapide et formidable essor, reste un "objet traductologique encore assez peu étudié ". Au commencement était le texte... Son analyse s'appuie sur la distinction fondamentale entre traduction théâtrale et surtitrage théâtral, la première produisant un texte destiné à la mise en scène, le second traduisant un texte issu de la mise en scène. Traduire une représentation, c'est travailler un texte fait de signes verbaux et non-verbaux, de codes ; un texte sans véritable matérialité physique mais incarné par les comédiens ; un texte en perpétuel mouvement, certes éphémère mais vivant. Dans l'idéal, le traducteur-surtitreur devrait donc collaborer étroitement au travail de création, de répétition et de représentation. Car les spécificités du texte " source ", comme celles du texte " cible ", génèrent des contraintes d'espace, de temps, de rythme qui auront à leur tour une incidence sur les choix traductifs : comment adapter, supprimer, réduire, condenser, transformer, sans affecter le sens à transmettre ? Faut-il sacrifier l'éventuelle qualité littéraire sur l'autel de la fonction utilitaire ? L'auteur s'interroge enfin sur les rapports entre scénographie et surtitrage. La réflexion sur le "texte-image " ne fait que commencer. Le mariage de la dimension linguistique et de l'élément graphique ouvre un espace de création nouveau. 
Bernard Péran poursuit ensuite sa réflexion avec Agnès Surbezy et tous deux s'intéressent au destinataire du surtitrage, parfois nommé "spectalecteur ". Selon son degré de compréhension de la langue source, le surtitrage lui sera absolument indispensable, partiellement nécessaire ou totalement superflu. Mais quelle sera l'expérience du spectateur sourd et malentendant, bénéficiant d'un double système de signes : la LSF et le surtitrage ? La LSF ne concernant véritablement qu'une fraction du public, qu'apporte-t-elle au spectateur entendant ? Et quels rapports entretiennent ces deux systèmes ? Peut-on parler de complicité et de complémentarité, au-delà des divergences fondamentales (le premier passe par un corps, le second est un texte-image) ? Oui, répondent les auteurs, illustrant leur propos d'exemples tirés de leur propre expérience avec la compagnie "Les Anachroniques". A. Surbezy et B. Péran plaident pour une pratique complémentaire des deux systèmes, ébauchant quelques pistes pour plus d'expressivité, d'interactivité et de lisibilité, notamment par un travail sur la dimension visuelle des surtitres : utilisation de polices typographiques ou de couleurs, de codes ou de pictogrammes. Dans un esprit d'enrichissement mutuel, de créativité et d'inventivité, tous les procédés doivent néanmoins rester au service de la " diffusion du théâtre en langue originale auprès du plus grand nombre ".

Dans un compte rendu de rencontres organisées par le Laboratoire Agôn-dramaturgies des arts de la scène, Alice Carré et Barbara Métais-Chastanier s'interrogent : "Où commence la dramaturgie ? " et proposent une réflexion sur les affinités entre le traducteur et le dramaturge, tous deux " arpenteurs de ce lieu de parcours entre la lettre et l'esprit ", " travaillant à des opérations de déplacements et de conversion ". Dans les rapports du premier à l'auteur, et du second au metteur en scène, que recouvrent les notions de "liberté " et de "fidélité " ? Peutêtre la " pratique d'un choix responsable ". Au-delà des affinités se dessine pourtant la singularité des pratiques et des positions de chacun, " dès lors que la scène entre dans le champ [des] possibles ". Le plateau devient alors ligne de partage. Mais la scène n'est pas l'unique finalité d'une traduction, qui peut être également, ou exclusivement, destinée à la publication. Dans ce cas, que publie-t-on ? Une "traduction de référence " ? Encore faudrait-il pouvoir établir les critères définissant cette notion, " tant les grilles de lecture évoluent avec les époques et les habitudes de traduction ". Quelles seraient les implications dramaturgiques de cette fameuse "traduction de référence " ? S'il existe un texte de référence, comment justifier une retraduction ? Où commence la fidélité ? Où s'arrête le traducteur? Où commence l'auteur?

"Écrire et traduire pour la scène : un même territoire du sensible ", répondent Marianne Ségol et Karin Serres, respectivement traductrice de théâtre jeunesse et autrice/traductrice, cofondatrices du LAB007, réseau de travail, d'expérimentation et de réflexion dans le théâtre contemporain européen pour la jeunesse. Elles présentent une expérience novatrice de traduction en duo (qui devient trio avec l'intervention active de l'auteur dans la phase finale de la traduction). À une première phase de travail séparé, permettant à chacune de trouver son " vocabulaire de fond ", succède un travail commun, mot par mot, dans "l'épaisseur " et " l'intimité " du langage. Vient ensuite un véritable jeu de déconstruction et reconstruction du 
texte, dans lequel chaque mot est pesé pour mieux en saisir le sous-texte et la vision de l'auteur. Celui-ci est alors invité à une séance de travail. Le témoignage enthousiaste d'Erik Uddenberg, auteur suédois, prouve l'intérêt théâtral et linguistique de l'expérience et ouvre de nouveaux horizons pour ce type de collaboration. Explorer le " territoire du sensible " entre un auteur et ses traducteurs peut se décliner sur une inversion du sens de traduction, ou l'association de l'écriture et de la traduction " dès les premiers mots d'un projet multilingue ". Inventive et créative, ouverte, dynamique et interactive, la démarche de M. Ségol et K. Serres est au plus près du cœur vivant du théâtre européen.

\section{Épilogue}

Le cahier hors dossier de ce numéro donne la parole à Jean-François Allain et David ar Rouz. Le premier propose de repenser la révision et plaide pour la relecture croisée. Pratiquée depuis plus de six ans dans le Service de la traduction française du Conseil de l'Europe, cette méthode offre de multiples avantages et s'avère un élément essentiel dans la mise en place d'une politique d'assurance qualité.

À partir de son questionnement initial sur les langues régionales (" facteur de division ou manière d'être ensemble? "), le second décline une profonde réflexion sur la notion de langue qui met la traduction au cœur de tout échange humain.

Enfin, Freddie Plassard nous offre un réjouissant compte rendu de lecture d'un ouvrage de Brice Matthieussent au titre prometteur, pour ne pas dire alléchant : Vengeance du traducteur.

Ai-je entendu "sublimation " ?

Bonne lecture!

g.begou@sfr.fr 